\title{
METODOLOGÍA PARA LA IDENTIFICACIÓN DE BARRERAS EN LOS PROCESOS DE CERTIFICACIÓN EN INOCUIDAD ALIMENTARIA: ESTUDIO DE CASO DE LA INDUSTRIA CÀRNICA DE BOVINO DE LA CIUDAD DE CÚCUTA.
}

\section{METHODOLOGY FOR THE IDENTIFICATION OF BARRIERS IN FOOD SAFETY CERTIFICATION PROCESSES: CASE STUDY OF THE BOVINE MEAT INDUSTRY IN THE CITY OF CÚCUTA.}

\author{
Naydú Judith Jácome Castilla ${ }^{1}$ \\ Eduardo Luis Jácome Castilla² \\ Erwin Hernando Jácome Castilla ${ }^{3}$ \\ Universidad Francisco de Paula Satander, \\ Colombia
}

\section{RESUMEN}

Las industrias alimentarias son organizaciones empresariales que deben garantizar en los procesos de producción la inocuidad de los alimentos, entendiéndose esto, como el cumplimiento de unas condiciones en la cadena de producción, almacenamiento y distribución que aseguren a los consumidores la ingesta de productos sin representar riesgos

$1 \quad$ Naydú Judith Jácome Castilla Universidad Francisco de Paula Satander Ocaña, Colombia ORCID https://orcid.org/0000-0001-9241-0522 njjacomec@ufpso.edu.co

2 Universidad Francisco de Paula Satander, Colombia. ORCID https://orcid.org/0000-0003-4958-9197 eduardoluisjc@ufps.edu.co

3 Universidad Francisco de Paula Satander, Colombia. ORCID https://orcid.org/0000-0003-1181-3552 erwinhernandojc@ufps.edu.co

para la salud humana. Sobre la base de estas consideraciones, es de vital importancia que la industria implemente procesos de certificaciones internacionales, que midan la calidad de sus procesos para el control integral de la inocuidad en las etapas de la cadena productiva. En esta orden de ideas, siendo el sector cárnico bovino la actividad comercial que más aporta al producto interno bruto $y$ a la generación de empleo en Colombia, el presente artículo, muestra los resultados de un proceso de investigación que buscaba analizar las barreras en la implementación de normas internacionales en la fabricación de productos cárnicos de la ciudad de Cúcuta. Desde los preceptos de establecidos por la International 
Organización for Standadization, con la norma "Garantía de la seguridad alimentaria", se estudiaron los principales impedimentos que presentan las empresas de Cúcuta en el cumplimiento de las condiciones para obtener la certificación internacional. La metodología aplicada se fundamentó en una investigación descriptiva con un enfoque mixto. Se diseño y aplicó un instrumento a las empresas de sector en la ciudad de Cúcuta, con el propósito de diagnosticar la situación actual, seguidamente mediante entrevistas se realizo una valoración de juicios de expertos, identificando la gestión empresarial, a nivel internacional y regional, aportando desde la experiencia los conocimientos necesarios en la implementación, mantenimiento y auditoria de sistema de gestión de calidad. Finalmente se correlaciono el jucio de expertos con el diagnostico, evaluando el nivel de cumplimiento, las principales barreras, el comportamiento del sector y el mejoramiento continuo para la fabricacion de alimentos 477 . inocuos.

\section{PALABRAS CLAVE}

1. Inocuidad alimentaria - 2. Sector cárnico - 3. Certificación internacional $\cdot$ 4. Cadena de producción $\bullet 5$. Mejoramiento continuo.

\section{ABSTRACT}

Food industries are business organizations that must guarantee food safety in production processes, understood as the fulfillment of conditions in the production, storage and distribution chain that ensure consumers can eat products without representing risks to human health. Based on these considerations, it is of vital importance that the industry implements international certification processes, which measure the quality of its processes for the integral control of safety in the stages of the production chain. In this order of ideas, being the bovine meat sector the commercial activity that contributes more to the gross domestic product and to the generation of employment in Colombia, the present article shows the results of a research process that sought to analyze the barriers in the implementation of international standards in the manufacture of meat products in the city of Cúcuta. From the precepts of established by the International Organization for Standadization, with the norm "Guarantee of the nourishing security", the main impediments that present/display the companies of Cúcuta in the fulfillment of the conditions to obtain the international certification were studied. The applied methodology was based on a descriptive research with a mixed approach. An instrument was designed and applied to the companies of the sector in the city of Cúcuta, with the purpose of diagnosing the current situation, then through interviews an evaluation of expert opinions was made, identifying the business management, at international and regional level, providing from experience the necessary knowledge in the implementation, maintenance and audit of quality management system. Finally, the expert's judgment was correlated with the diagnosis, evaluating the level of compliance, the main barriers, the behavior of the sector and the continuous improvement for the manufacture of safe food.

\section{KEYWORDS}

1. Food Safety 2. Meat Sector 3. International Certificatión 4. Productión Chain 5. Continuos Improvement

\section{INTRODUCCIÓN}

En Colombia el sector cárnico, esta representado por las actividades desde la cría de animales hasta la transformación de materia prima de carnes para la generación de productos terminados para el consumo humano; es así como, la estructura reproductiva de la industria cárnica inicia desde la cría y engorde del ganado vacuno, transporte, sacrificio, corte, congelación, comercialización para la 
elaboración de productos cárnicos como carnes embutidas, arregladas y frías. (Departamento Nacional de Planeación, 2004). En este sentido, al referirse de la industria cárnica la legislación Colombiana en el decreto 1500 de 2007 la reconoce como derivados cárnicos (Ministerio de Salud, 2007), creando mediante esta ley, el sistema oficial, vigilancia y control de la carne, productos cárnicos y derivados para el consumo humano y los requisitos sanitarios y de inocuidad que se deben cumplir para la comercialización y expendio de estos productos.

En cuanto a la importancia de la industria ganadera, esta aporta el $1.6 \%$ del Producto Interno de Bruto Nacional (Coneo, 2019), con una producción de 933.000 toneladas equivalentes a 4.1 millones de bovinos (Portafolio, 2020), representando esta industria un impacto alto en la producción nacional como fuente de ingresos y de cobertura al mercado nacional e internacional, el cual para los dos primeros meses del año 2020, ya había exportado más de 4.5 toneladas de carne a ocho país, obteniendo ganancias cercanas a los 18 millones de dólares (Asmar, 2020), generadas por la estrategia de diplomacia sanitaria que busca la confianza de los mercados a partir de la certificación otorgada por la Autoridad Sanitaria Internacional IOE, donde clasifican a Colombia como un país libre de fiebre Aftosa. (Insituto Agropecuario Colombiano, 2020)

El tejido empresarial del sector cárnico en Colombia posee socios estratégicos de la cadena de producción a nivel nacional con empresas que se dedican a la producción, transformación y comercialización, los cuales están concentrados por regiones según el conteo de ejemplares bovinos, siendo la región 1 los departamentos entre 1 millón y 2,5 millones de ejemplares bovinos (Córdoba, Casanare, Meta, Cesar, Santander, Caquetá, Magdalena, Cundinamarca y Arauca); la región 2 los departamentos entre 200.000 y 800.00 ejemplares bovinos (Bolívar,
Sucre, Boyacá, Tolima, Huila, Valle del Cauca, Norte de Santander, Nariño, La Guajira, Caldas, Cauca, Vichada y Atlántico) y la región 3 los departamentos menos de 200.000 ejemplares bovinos (Putumayo, Chocó, Quindío, Guaviare, Risaralda, Amazonas, Guainía, San Andrés y Providencia y Vaupés) (Procolombia, 2015). Norte de Santander en producción de carne se encuentra ubicada en la región 2 , con 247.898 semovientes registrando un aumento de $7.8 \%$ en producción del año 2018 al 2019; cifras que reflejan que el gremio ganadero aporta de forma considerable la materia prima utilizada para la transformación de la carne en productos cárnicos. En este sentido, y teniendo en cuenta que la industria cárnica en su proceso productivo en la etapa de transformación a la etapa de envasado hace referencia a la transformación de materia prima carne de canal a carnes frías y embutidos como de productos derivados, se constituye un eslabón de la cadena alimentaria en Colombia y en el Departamento, es preciso acotar la importancia de la cadena de producción no solo como indicadores que generan guanacias, sino también como fuente de consumo en las necesidades alimentarias de la región.

En este contexto, al referirse de cadena alimentaria la legislación colombiana desde el Ministerio de la protección social crea un sistema de vigilancia y control para el expendio de productos para el consumo humano y la inocuidad como requisitos básicos que deben cumplir las organizaciones que ejercen actividades relacionadas con alimentos; es así como nuestro país ha venido actualizando y mejorando las normas de inocuidad en la industria alimentaria con el decreto 1500 de 2007 en el cual expide los requisitos sanitarios y de inocuidad que se deben cumplir en su producción primaria y el decreto 3075 de 1997; que Dicta las normas que deben cumplir los establecimientos encargados de la transformación de alimentos, en él se establecen las buenas prácticas de manufactura 
(BPM). Igualmente, estas organizaciones deben aprovechar las condiciones de la industria para impulsar a mercados internacionales soportados en los tratados de libre comercio para el beneficio de los sectores más productivos, invirtiendo todos los esfuerzos hacia la tecnificación y desarrollo de las industrias para mejorar el acceso a mercados internacionales de los productos colombianos.

En este sentido y partiendo de la base que el mejoramiento de las industrias en términos de calidad en los procesos de producción es medido por los estándares internacionales por la International Organization for Standadization, con la norma "Garantía de la seguridad alimentaria y de acuerdo a la importancia de la industria cárnica en Colombia la cual genera 28. 222 empleos directos (Departamento Nacional de Planeación, 2020) siendo generadora de desarrollo en Norte de Santander, el presente estudio realizó un análisis de los principales impedimentos que presentan las empresas dedicadas a la fabricación de derivados cárnicos de la ciudad de Cúcuta, en el cumplimiento de las condiciones para obtener la certificación internacional.

\section{MARCO TEÓRICO}

Para la construcción y desarrollo de la investigación realizada se tomaron las teorías y conceptos de diferentes autores sobre la industria cárnica, marco legislativo de inocuidad de los alimentos, y certificaciones internacionales establecidas por la International Organización for Standadization.

\section{a. Cadena Productiva de la Industria}

\section{Cárnica.}

En Colombia el sector cárnico se compone por un proceso productivo que comprende dos procesos, el primero hace referencia a un proceso pecuario con la reproducción, cría, levante, engorde y comercialización de ganado en pie, que agrupa las actividades asistencia técnica, mano de obra, alistamiento de materiales, terrenos, medicamentos, alimentos e insumos necesarios para la producción Ganado bovino en pie, ganado porcino en pie y aves de corral. (Nieto \& Ramirez, 2018)

Con la materia prima generada del proceso pecuario, se origina el segundo proceso denominado como industrial, el cual toma el ganado bovino y porcino en pie y las aves de corral para transformarlos, prepararlos y envasarlos para la obtención de productos derivados cárnicos como carnes frías, embutidos y huevos. (Departamento Nacional de Planeación, 2018) Siendo el sector cárnico bovino el segundo en consumo per cápita interno de 18,6 kilogramos anuales. (Fedegan, 2019); convirtiéndose esto en retos importantes en cuanto competitividad y calidad, incitando a las empresas que se dedican a estas actividades económicas, a que se generen procesos productivos que modifiquen el mal estado de la cadena productiva en Colombia. (Guarin, 2008)

Con referencia a lo anterior y teniendo en cuenta la importancia de consumo per cápita la Federación Colombiana de Ganaderos en el programa de transformación productiva describe en el sector cárnico bovino como producción primaria, Frigoríficos, industria cárnica y distribución y venta, tal como se ilustra en la Figura 1. 


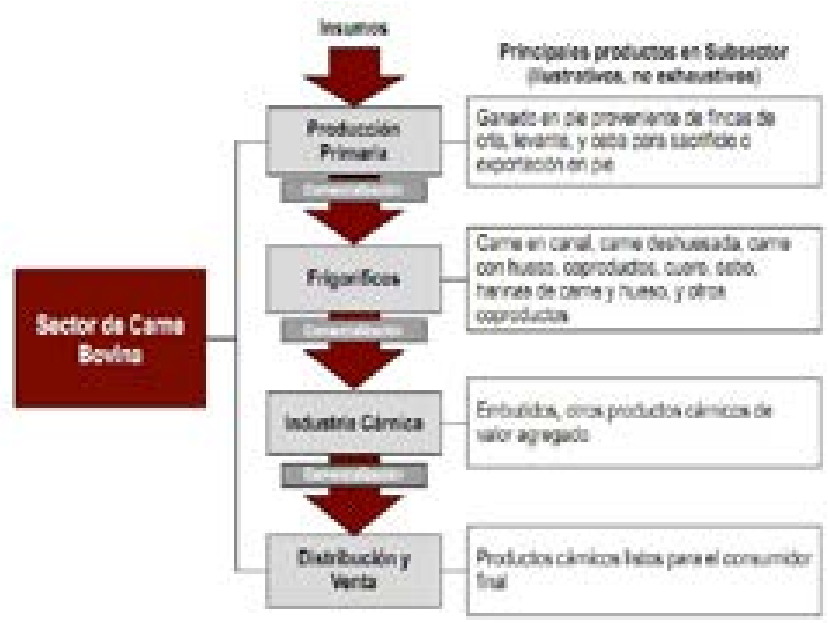

Figura 1. Sector de Carne Bovina

Fuente (Miniserio de Agricultura y Desarrollo Rural, 2010)

\section{b. El sector cárnico en cifras}

Un sector económico aporta desarrollo regional en términos de producción, empleos generados y exportaciones. Para el sector cárnico en Colombia la participación en producto interno bruto representa el $24,6 \%$ de la producción nacional, con un consumo por habitante de 18.2 kilogramos anuales; generando para el año 2018 aproximadamente 810.000 empleos en el sector de carne bovina. (Gobierno de Colombia, 2019). En cuanto a la distribución geográfica en inventario bovino en Colombia los principales departamentos según censo bovino 2018 son Antioquia, Córdoba, Casanare, Meta y Caquetá, como se ilustra en la figura 2.

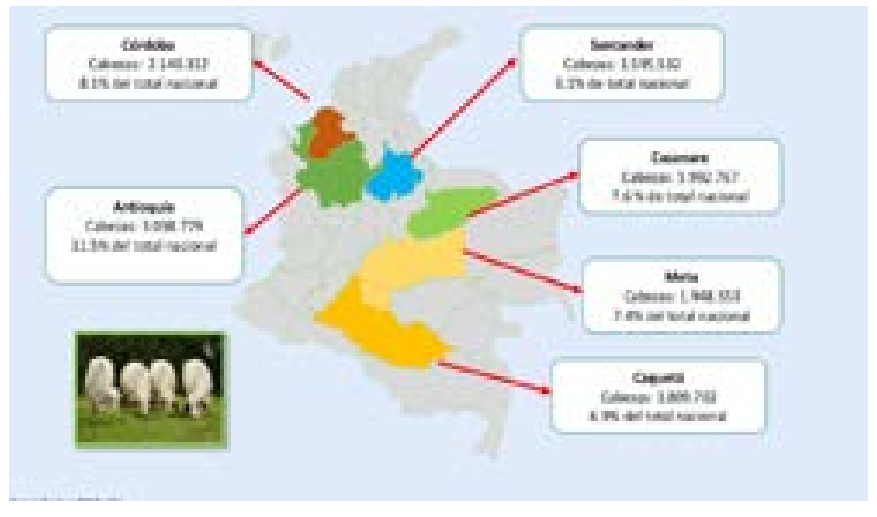

Figura 2. Principales zonas productoras de ganado de carne Fuente (Ministerio de Agricultura, 2019)

En este orden de ideas, al referirnos al mercado internacional el sector de carne Bovina de los años 2013, presentó una disminución en el nivel de exportaciones, que posteriormente se ha visto recuperada por la certificación otorgada al país como zona libre de aftosa, como se observa en la Figura 3, siendo preciso que para fortalecer el crecimiento del mercado internacional, se 
debe aprovechar los tratados de libre comercio que beneficia el sector en proceso de apertura de mercados para los productos derivados de carne bovina.

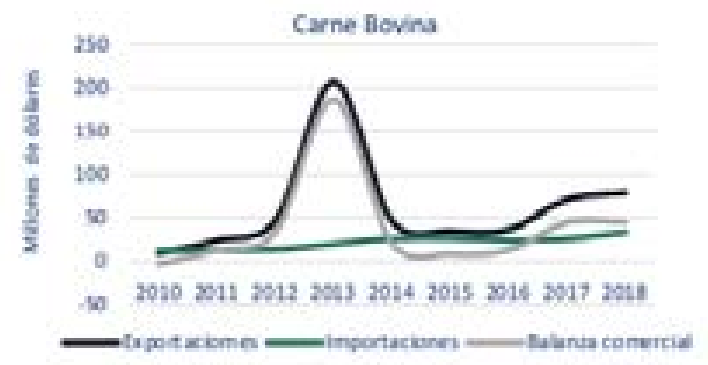

Figura 3. Comercio exterior del sector cárnico

Fuente (Gobierno de Colombia, 2019)

\section{c. Marco legal para la seguridad} alimentaria e inocuidad de los alimentos.

La seguridad alimentaria e inocuidad de alimentos, en el país constituyen elementos necesarios para el aseguramiento en los procesos productivos de las industrias cárnicas, presenta referentes normativos que reglamentan para estas actividades para la vigilancia y control de la actividad económica. En la siguiente figura se caracterizó cronológicamente la normatividad nacional , con el propósito de que las entidades aborden la legislación de este referente legal realizado en la investigación.

Tabla 1. Matriz reglamentaciones orden nacional.

\begin{tabular}{|c|c|c|}
\hline NORMATIVIDAD & DESCRIPCION & ENTIDAD \\
\hline Decreto 1500 de 2007 & $\begin{array}{l}\text { Por el cual se establece } \\
\text { el reglamento técnico a través del } \\
\text { cual se crea el Sistema Oficial de } \\
\text { Inspección, Vigilancia y Control } \\
\text { de la Carne, Productos Cárnicos } \\
\text { Comestibles y Derivados Cárnicos, } \\
\text { destinados para el Consumo } \\
\text { Humano y los requisitos sanitarios } \\
\text { y de inocuidad que se deben } \\
\text { cumplir en su producción primaria, } \\
\text { beneficio, desposte, desprese, } \\
\text { procesamiento, almacenamiento, } \\
\text { transporte, comercialización, } \\
\text { expendio, importación o } \\
\text { exportación. (Ministerio de } \\
\text { Protección Social, 2007) }\end{array}$ & $\begin{array}{l}\text { Emitida por: Ministerio del Interior } \\
\text { y de Justicia de la Republica. } \\
\text { Entidad responsable: INVIMA }\end{array}$ \\
\hline
\end{tabular}




\begin{tabular}{|c|c|c|}
\hline Decreto 3075 de 1997 (BPM) & $\begin{array}{l}\text { Dicta las normas que } \\
\text { deben cumplir los establecimientos } \\
\text { encargados de la transformación de } \\
\text { alimentos, en él se establecen las } \\
\text { buenas prácticas de manufactura. } \\
\text { regula todas las actividades que } \\
\text { puedan generar factores de riesgo } \\
\text { por el consumo de alimentos. } \\
\text { (República, 1997) }\end{array}$ & $\begin{array}{l}\text { Emitida por: presidencia de la } \\
\text { Republica. } \\
\text { Entidad responsable: INVIMA }\end{array}$ \\
\hline Decreto 2162 de 1983 & $\begin{array}{l}\text { Por el cual se reglamenta } \\
\text { parcialmente el título V de la ley } 09 \\
\text { de } 1979 \text {, en cuanto a producción, } \\
\text { procesamiento, transpor1e y } \\
\text { expendio de los productos cárnicos } \\
\text { procesados }\end{array}$ & $\begin{array}{l}\text { Emitida por: presidencia de la } \\
\text { Republica. } \\
\text { Entidad responsable: INVIMA }\end{array}$ \\
\hline Decreto 2380 de 2009 & $\begin{array}{l}\text { Por el cual se modifican } \\
\text { los Decretos } 1500 \text { de } 2007 \text { y } \\
2965 \text { de } 2008 \text { y se dictan otras } \\
\text { disposiciones. (Ministerio de } \\
\text { Protección Social, 2009) }\end{array}$ & $\begin{array}{l}\text { Emitida por: presidencia de la } \\
\text { Republica. } \\
\text { Entidad responsable: INVIMA }\end{array}$ \\
\hline Resolución 2905 de 2007 & $\begin{array}{l}\text { Por la cual se establece } \\
\text { el reglamento técnico sobre los } \\
\text { requisitos sanitarios y de inocuidad } \\
\text { de la carne y productos cárnicos } \\
\text { comestibles de las especies } \\
\text { bovina y bufalina destinados } \\
\text { para el consumo humano y las } \\
\text { disposiciones para su beneficio, } \\
\text { desposte, almacenamiento, } \\
\text { comercialización, expendio, } \\
\text { transporte, importación o } \\
\text { exportación. ( Ministerio de la } \\
\text { Protección Social, 2007) }\end{array}$ & $\begin{array}{l}\text { Emitida por: Ministerio de la } \\
\text { protección social } \\
\text { Entidad responsable: INVIMA }\end{array}$ \\
\hline Resolución 2674 del 2013 & $\begin{array}{l}\text { Por la cual se reglamenta } \\
\text { el artículo } 126 \text { del Decreto } \\
\text { Ley } 019 \text { de } 2012 \text { y se dictan } \\
\text { otras disposiciones, en temas } \\
\text { relacionados con vigilancia y } \\
\text { control de alimentos en cuanto a los } \\
\text { requisitos para el registro sanitario. } \\
\text { (Ministerio de la Protección Social, } \\
2013 \text { ) }\end{array}$ & $\begin{array}{l}\text { Emitida por: Ministerio de Salud y } \\
\text { protección social. } \\
\text { Entidad responsable: INVIMA }\end{array}$ \\
\hline
\end{tabular}


Una segunda matriz donde se describe la normatividad internacional de voluntario cumplimiento, en cuanto a la calidad de los procesos y la inocuidad alimentaria.

Tabla 2. Matriz normatividad para acceso a certificaciones internacionales.

\begin{tabular}{|c|c|c|}
\hline NORMATIVIDAD & DESCRIPCION & ENTIDAD \\
\hline Norma HACCP & $\begin{array}{l}\text { Se utilizará como guía los } \\
\text { conceptos dictados en esta norma, } \\
\text { los } 7 \text { principios que enmarcan } \\
\text { el control de la inocuidad de los } \\
\text { alimentos, los cuales ayudaran } \\
\text { como enlace entre las normas BPM } \\
\text { y la norma ISO 22000. (Carro \& } \\
\text { Gonzalez) }\end{array}$ & Voluntario cumplimiento \\
\hline Norma ISO 22000:2018 & $\begin{array}{l}\text { Esta norma específica los } \\
\text { requisitos para un sistema de } \\
\text { gestión de inocuidad de los } \\
\text { alimentos en la cadena alimentaria } \\
\text { cuando una organización necesita } \\
\text { demostrar su capacidad para } \\
\text { controlar los peligros relacionados } \\
\text { con la inocuidad de los alimentos } \\
\text { con el objeto de asegurar que el } \\
\text { alimento es inocuo en el momento } \\
\text { del consumo humano" (ISO, 2018) }\end{array}$ & $\begin{array}{l}\text { Voluntario cumplimiento } \\
\text { Emitida por: organización } \\
\text { internación de normalización ISO }\end{array}$ \\
\hline Norma ISO 9001:2015 & $\begin{array}{l}\text { "Especifica los requisitos para } \\
\text { un sistema de gestión de la } \\
\text { calidad cuando una organización: } \\
\text { necesita demostrar su capacidad } \\
\text { para proporcionar regularmente } \\
\text { sus productos y servicios que } \\
\text { satisfagan al cliente y los legales } \\
\text { y reglamentarios aplicables". (Iso, } \\
2015 \text { ) }\end{array}$ & $\begin{array}{l}\text { Voluntario cumplimiento } \\
\text { Emitida por: organización } \\
\text { internación de normalización ISO }\end{array}$ \\
\hline
\end{tabular}

\section{METODOLOGÍA O PROCEDIMIENTOS}

De acuerdo con la investigación planteada y las características del estudio, la metodología utilizada fue descriptivo (Hernández, 1998). Es así como, para establecer el diagnostico de la industria cárnica se aplicaron métodos cuantitativos con recolección de información de fuentes primarias mediante la aplicación de entrevistas; igualmente, se emplearon datos que permitieron determinar el nivel de cumplimiento de la reglamentación nacional y las normas internacionales que garantizan la inocuidad de los alimentos. Por otra parte, para el desarrollo del procyecto (Lucio, 2003), que permitieron determinar los principales impedimentos por los 
cuales las empresas de la industria cárnica no se certifican en inocuidad alimentaria.

El método utilizado es de tipo descriptivo (HERNANDEZ SAMPIERI, 2003), dado que se analizaron los impedimentos más sobresalientes por los cuales las organizaciones no cumplen con todos los aspectos requeridos para la obtención de certificados. La muestra considerada para el estudio, se definió como el subconjunto finito y representativo de la población (Arias, 2006), siendo a conveniencia (Otzen \& Manterola, 2017), seleccionando las empresas de la industria cárnica que accedieron a la evaluación de los procesos de sus organizaciones por parte de los investigadores. En este orden de ideas, el proyecto estructuró en su metodología 4 fases para el diseño del método y la consecución de los resultados, denominado como momentos de la investigación.

Tabla 3. Matriz momentos de la Investigación

\begin{tabular}{|c|c|}
\hline Momentos & Descripción \\
\hline Momento 1 & $\begin{array}{c}\text { Diseño del anteproyecto, planteamiento de la problemática de la } \\
\text { investigación, objetivos, metodología, diseño de instrumentos. }\end{array}$ \\
\hline Momento 2 & $\begin{array}{c}\text { Aplicación del instrumento y análisis de la información cuantitativa } \\
\text { que permitió diagnosticar la industria cárnica de la ciudad de Cúcuta. }\end{array}$ \\
\hline Momento 3 & $\begin{array}{l}\text { Panel de expertos, encuentro con especialistas calidad y } \\
\text { autoridades empresariales y económicas de la ciudad. }\end{array}$ \\
\hline Momento 4 & $\begin{array}{c}\text { Análisis de correlación de los instrumentos aplicados y paneles } \\
\text { de expertos, que sirvieron para determinar los principales impedimentos } \\
\text { de la industria en la ciudad. }\end{array}$ \\
\hline
\end{tabular}

\section{RESULTADOS, ANÁLISIS E INTERPRETACIÓN}

En esta sección se presenta los resultados del estudio; realizando un análisis a las respuestas dadas por los empresarios y a las experiencias aportadas por los expertos y autoridades económicas de la ciudad. En este sentido, en primer lugar y partiendo del postulado que "el resultado de los procesos de investigación estan relacionados con la organización y el funcionamiento de las empresas, que permite determinar y evaluar las relaciones causaefectos de los problemas detectados y dar solucion integral a los mismos" (Velasquez, 2009).

\section{a. Diagnóstico de la industria cárnica de las empresas de Cúcuta.}

Se realizó un diagnóstico de la industria cárnica en la ciudad de Cúcuta, para establecer el contexto sobre el nivel de cumplimiento en la norma de sistema de gestión de inocuidad alimentaria, bajo el precepto que la investigación no mide el cumplimiento los aspectos legales como decretos o resoluciones en cuanto a la inocuidad alimentaria de los procesos de la industria. Como una idea inicial, se pudo observar que las empresas tienen temor acogerse a las normas internacionales por razones como costos en los procesos, sometimiento auditorias; expresando a su vez que estas implementaciones no generan valor agregado y solo sirven para mostrar falencias de inocuidad que poseen la empresas; en este caso particular las auditorias se toman para encontrar los motivos que impiden alcanzar las certificaciones (de voluntaria adopción), y mejorar los procesos progresivamente de acuerdo a las capacidades de cada empresa; dado que estas normas no tienen implicaciones legales y las organizaciones las adoptan de 
manera voluntaria para el acceso a los mercados internacionales.

Es así como, desde el primer acercamiento con las organizaciones, se pudo determinar que ninguna de las empresas de la industria cárnica se encuentra certificadas con normas internacionales de inocuidad alimentaria, para lo cual se estableció la aplicación de un instrumento de 200 preguntas, con el propósito de evaluar que aspectos cumplen y cuales son las debilidades para fortalecer para que las organizaciones puedan logran procesos de calidad en la fabricación de sus productos. El primer aspecto hace referencia al sistema de gestión de los alimentos; en cuanto a los requisitos generales y documentación necesaria para la creación del sistema; esto con el fin que la organización establezca, implemente, mantenga, actualice y mejore continuamente el sistema. (INCONTEC, 2019).

Partiendo de esta idea, se observó que en la industria cárnica ninguna de las empresas cuenta con los aspectos necesarios para la implementación de la normatividad, dado que no tienen definido el alcance, la política de inocuidad y los objetivos; sin embargo, es preciso descartar que tienen plenamente establecidos los peligros de inocuidad de los alimentos; para lo cual las empresas tienen "identificados, evaluados y controlados de manera que los productos de la organización, no perjudiquen directa o indirectamente al consumidor" (ICONTEC, 2005)

\section{SISTEMA DE GESTION DE ALIMENTOS INDUSTRIA CÁRNICA CMPRESAS CUCUTA}

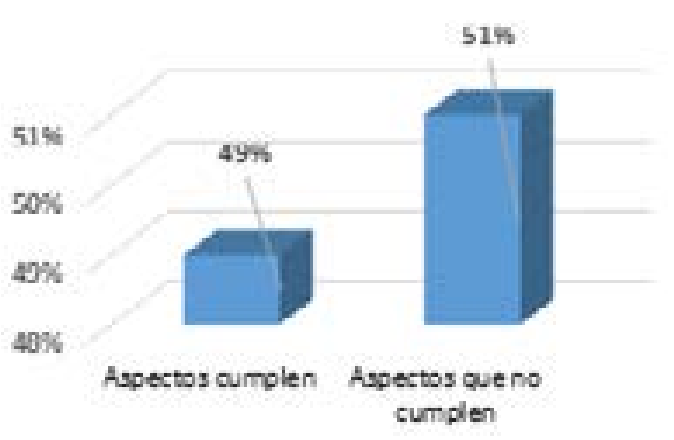

Figura 4. Sistema de Gestión de Alimentos Industria Cárnica de las empresas de Cúcuta

Fuente: Elaborado por los autores

Asimismo, se evidenció que tienen comunicación fluida con toda la cadena alimentaria, sin embargo, no diligencian los formatos, aunque visto desde la normatividad legal, las organizaciones cumplen con los registros y requisitos legales en cuanto a la documentación, estos no están debidamente establecidos en procedimientos reflejando falencias en el control de los documentos.

En cuanto al liderazgo, la alta dirección debe demostrar liderazgo y compromisos con respecto al Sistema de Inocuidad Alimentaria, para lo cual el estudio determinó la existencia de una política y como son las directrices de la gerencia para comunicar la importancia de una gestión eficaz de la inocuidad de los alimentos y el cumplimiento de los requisitos del sistema. (INCONTEC, 2019). Observando que la indsutria cárnica en su mayoria no cumple los aspectos básicos que deben seguir la alta dirección. 


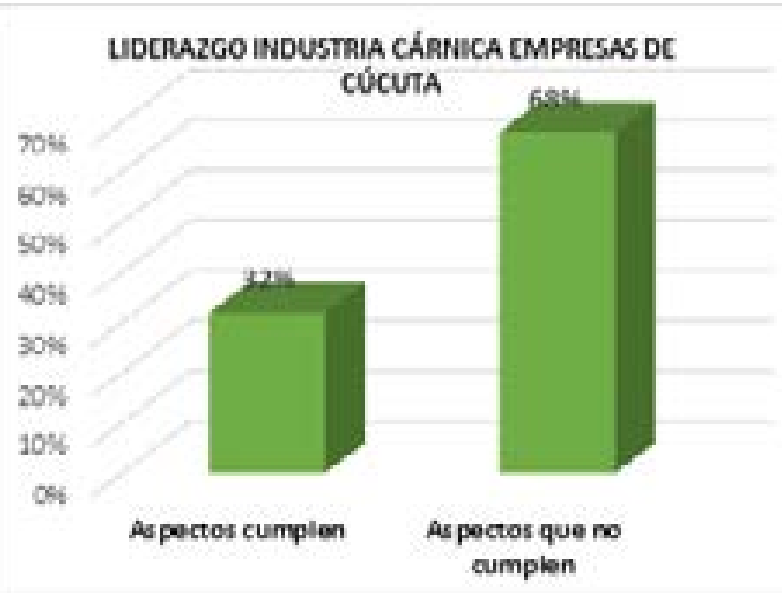

Figura 5. Liderazgo Industria Cárnica de las empresas de Cúcuta

Fuente: Elaborado por los autores

Es asi como, los administradores del sistema deben ser lideres que establezcan los propositos y orientaciones de la organización, con la responsabilidad de crear y mantener un ambiente interno en el cual sus partes ineresadas se involucren totalmente con el logro de los objetivos. (Hermann Keyserling, 2010). Bajo la premisa anterior, al encuestar a los lideres de la industria de las organizaciones estudiadas, estos coincidieron en el mejoramiento de la inocuidad de los procesos para ofrecer productos sanos a los consumidores; sin embargo se observó que no tienen establecida una politica de inocuidad que apoye la producción.

El liderazgo mide la importancia de una gestión eficaz de la inocuidad de los alimentos y cumplimiento de los requisitos del sistema establecidos por la ley y los previamente acordados con los clientes. (INCONTEC, 2019) Para lo cual, se encontró que las organizaciones presentan una comunicación fluida con la cadena alimentaria y designan personas para realizar estas actividades, sin embargo no existe algún documento que evidencie como se realiza esta selección. Igualmente, se puede precisar que las comunicaciones internas se realizan por medio de capacitaciones concernientes a la inocuidad del producto. Por otra parte, en cuanto a las situaciones de emergencia, accidentes y retiradas del producto, las empresas estan en la capacidad y poseen los formatos y procedimientos para actuar ante estas eventualidades, asumiento la responsabilidad de la dirección en casos en que los productos puedan causar problemas a los consumidores. Dicho lo anterior, se puede concluir que la dirección de las organizaciones poseen barreras altas por superar, debido a la falta de compromiso.

Continuando con los aspectos analizados y teniendo en cuenta que para el mantenimiento de un sistema de gestión es necesario que las organizaciones determinen y proporcionen los recursos necesarios para el establecimiento, implementación, mantenimiento, actualización y mejora continua del sistema, se analizó los recursos que poseen estas en cuanto a infraestructura y personal. En este sentido, las organizaciones no cuentan con un equipo de inocuidad, sin embargo tienen un personal capacitado para la elaboración de productos inocuos. Con respecto al cumplimiento de los recursos necesarios para el mejoramiento de los procesos la industria no cumple con la totalidad; no obstante las empresas buscan recursos para invertir en el mejoramiento de sus instalaciones, muestra de ello son los aumentos reflejados en los historicos de producción. Cabe agregar, como aspectos importantes de las empresas el cumplimiento de la formación exigida por la ley para el personal encargado de las labores de inocuidad de los procesos, pese a esto presentan falencias en la formación y la competencia del personal. Por estas razones enunciadas, se puede concluir que los apoyos se convierten en una barrera para la certificación, dado que necesitan inversiones y prestamos para el mejoramiento continuo.

Para la planificación y control operacional de productos inocuos, se proyectan todos 
los aspectos necesarios para la elaboración de alimentos que no signifiquen ningún tipo de peligro para el consumo humano, aquí se desarrollan todos los aspectos técnicos que conllevan asegurar procesos correctos y proteger de contaminación las materias primas y los productos terminados, así como garantizar que las instalaciones los ingredientes y los materiales no afecten la inocuidad. Por esta razón y para contar con procesos de alta calidad en la industria de alimentos se debe implementar programas y prerrequisitos operacionales estos ayudan a controlar los peligros existentes en la producción. Dados los requerimientos de fabricación de un producto apto para el consumo humano, se observó que en este aspecto las organizaciones presentan un nivel de cumplimiento similar a los anteriormente mencionados, según lo muestra la figura 5 , la empresas cumplen en bajo nivel los aspectos, realizando un esfuerzo en la industria cárnica por la obtención de productos inocuos y por el mejoramiento de los procesos relacionados con la inocuidad del producto, a pesar de ello hace falta mayor inversión y preocupación

\section{PI ANIFICACIÓN Y CONTROI OPIRACIONAI INDUSIKIA CÁKNICA EMPKESAS UE CUCUIA}

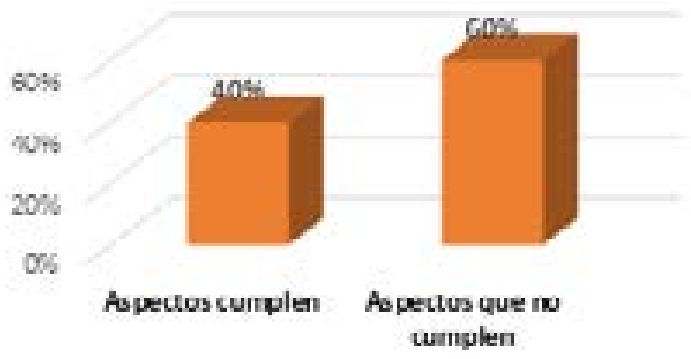

Figura 5. Planificación y control operacional Industria Cárnica de las empresas de Cúcuta

Fuente: Elaborado por los autores

Los productos que se llevan a cabo en este sector son inocuos y muestra de ello, es el crecimiento de las ventas y el cumplimiento de la Ley Colombiana. En atención a esto mediante la realización del estudio se buscaba identificar las principales barreas para mejorar la calidad con la que se produce, para así crecer como industria y convertirse en un sector competitivo. (Heras, Arana, Camison, Casadesus, \& Martiarena, 2008)

De acuerdo con lo anterior, en este aspecto se analizó los programas prerrequisitos, encontrando que no se están implementado en todos los sistemas de gestión, aunque consideran los programas y disposiciones en cuanto a técnicas de espacio, suministros de agua, aire y energía las instalaciones son pequeñas, haciendo esfuerzos considerables para reformarlas a medida que las exigencias van aumentando. Igualmente, las organizaciones cumplen con los programas de limpieza y desinfección, control de plagas, higiene personal lo que hace mostrar el compromiso con los peligros que pueden afectar la inocuidad de los productos.

Con respecto a las descripciones se presenta una variabilidad en la industria, dado que en cuanto a los requisitos referentes al proceso de producción tales como: ingredientes que hacen parte del producto, condiciones de almacenamiento y métodos de producción, no existe un patrón definido que haga posible el análisis como conjunto, se cumplen algunos aspectos y otro se pasan por alto, observando que no tienen todos los elementos documentados que hacen posible un mejor historial de producción, que permita conservar todas las características ideales de las materias primas y de los productos terminados y el uso previsto.

En cuanto a la producción se puede acotar, que las empresas poseen diagramas de flujo (Heizer, 2007), mostrando la interacción de las etapas del proceso con la carencia de la especificación de estos, por lo que se deben mejorar y completar estos diagramas para asumir una precisión de las etapas y así tener una comunicación clara con los trabajadores 
que permita el conocimiento y detalle del proceso productivo. Así mismo, para lograr altos niveles de inocuidad alimentaria, entendida esta como "La calidad e inocuidad se refieren a las características de los alimentos, que garantizan que estos sean aptos para el consumo humano y que exigen el cumplimiento de una serie de condiciones y medidas necesarias, durante la cadena agroalimentaria, hasta el consumo y el aprovechamiento de estos, asegurando que una vez ingeridos no representen un riesgo (biológico, físico y/o químico) que menoscabe la salud" (Ortiz \& Martinez, 2011); es muy importante realizar una buena identificación de peligros, dado que de esta actividad parten los controles que se realizaran a la producción; sin embargo, las empresas están dejando de lado ejecutar estas actividades, por consiguiente, solo se limitan a atacar esos peligros que los han afectado en anteriores ocasiones

Las falencias en este aspecto abarcan también la identificación de análisis de peligros en toda la cadena productiva tanto en los eslabones que anteceden a la transformación y las que preceden a la misma. Por tal motivo es importante que se realice esta acción porque de allí se puede determinar el nivel mínimo aceptable, aspecto que las empresas no tienen en cuenta en sus procesos, solo basta con el cumplimiento legal.

De acuerdo con lo anterior, el paso siguiente para las empresas es realizar la evaluación de peligros (Bryan , 1992), este ítem las empresas no lo cumplen y de allí parte una mala elección de los controles para la inocuidad de los alimentos puesto que con ello se busca prevenir eliminar o reducir estos peligros. Cabe resaltar que las medidas control seleccionadas por las empresas, no presenta un riguroso análisis y tampoco se mide la eficacia de los controles seleccionados. Estas organizaciones no tienen programas prerrequisitos operativos y en cuanto a los planes HACCP están incompletos, dado que se encuentran documentados generalmente para dos productos los cuales no se aplican o implementan en los procesos.

Existen establecidos mecanismos que hacen posible la identificación de lotes de producción la materias primas e ingredientes utilizados en los procesos, pero las organizaciones no tienen sistemas de trazabilidad que les permita tener el control total, resulta oportuno aclarar que las empresas están en la tarea de implementación de estos procedimientos en la actualidad. Para el tratamiento de los productos no conformes la norma indica la superación de los limites críticos (Organización Panamericana de Salud), más las empresas no tratan sus productos no conformes de esta manera, tiene dispuestos otros aspectos conforme con el cumplimiento legal, asegurándose que los productos no inocuos no regresen a la cadena alimentaria, aunque no se cuente con procedimientos documentados para tratar estos productos. De esta manera se puede concluir que se encuentran barreras técnicas que hacen mayor la dificultad de someterse a un proceso de certificación de la norma indicando que son bastante amplios los rangos para el cumplimiento de los aspectos que hacen blindar a la producción con la protección necesaria y las disposiciones correctas para obtener productos inocuos.

Para finalizar el análisis, se estableció el cumplimiento de los aspectos de mejora, la validación que deben aplicar a todos los controles, que las organizaciones reconocieron y aplicaron en todas las etapas del proceso productivo. Igualmente, la verificación que se debe realizar mediante auditorias internas e inspecciones realizadas por entes estatales; y por último la mejora continua y la actualización del sistema. 


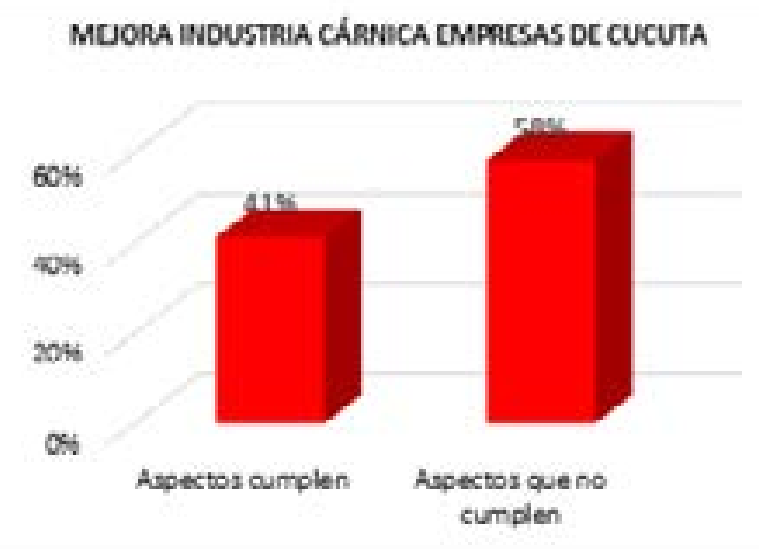

Figura 6. Mejora Industria Cárnica de las empresas de Cúcuta

Fuente: Elaborado por los autores

En este aspecto, las empresas calibran todos sus equipos con el fin de obtener valores confiables que representen a las empresas tranquilidad, al observar el cumplimiento pleno, la figura 6 ilustra que las organizaciones en un porcentaje del $58 \%$ no cumplen con los requisitos para el total cumplimiento del aspecto, sin embargo, cuenta con los registros necesarios, que hacen ver una fortaleza en este en las organizaciones encuestadas, pese a que no se cuentan con programas de auditorías internas que permitan una revisión clara sobre los aspectos que fallan en la organización. No obstante, existe un interés generalizado por la mejora continua de los procesos que realizan, pero deben tener en cuenta la implementación de normas de calidad que ayuden a fortalecer el sector de la industria cárnica de la ciudad de Cúcuta. Así mismo se deben ejecutar auditorias porque de allí parte el mejoramiento de los procesos; de esta manera se hace evidente que las organizaciones pertenecientes al sector cárnico de la ciudad de Cúcuta tienen barreras para el cumplimiento de este aspecto. b. Foro de expertos: Una mirada desde la experiencia académica, empresarial y autoridades económicas de la ciudad.

El Foro de expertos constituyó un eje primordial en los resultados de la investigación, dado que se buscaba identificar desde la óptica de los empresarios y los agentes económicos las principales barreras que se presentan al momento de la implementación y certificación en sistemas de gestión. El foro desde la perspectiva del profesor Fidel Aragón Franco, asesor de calidad con experiencia en empresas a nivel de latinoamerica, en sectores industriales y servicios, académico de varios paises; buscaba conocer desde una optica internacional las principales barreras que ha identificado el experto con la implementación de normas internacionales de inocuidad alimentaria.

Desde los postulados anteriores, la participación del asesor y la interacción con el público conformado por empresarios, docentes y estudiantes de la entidad académica en la cual se desarrolló el evento, se puede concluir que a nivel internacional, las empresas latinoamericanas presentan miedos, temores y resistencia al cambio, generando mayores costos para la organizaciones, ocasionados por las falencias en los procesos productivos que implican pérdidas económicas, afectando la maximización de las utilidades.

Desde la óptica regional, el académico José Barrera Gómez, docente que desarrolla los procesos de calidad de la Universidad Simón Bolívar sede Cúcuta, y quien cuenta con experiencia en certificaciones internacionales de varias organizaciones de la región; aportó al foro las experiencias de los empresarios a nivel regional sobre las practicas y obstáculos en la implementación de certificaciones internacionales. 
De acuerdo a lo planteado en su intervención por el docente José Barrera, se puede inferir que a nivel regional, se presentan los mismos obstáculos de las empresas latinoamericanas, reflejada por el desconocimiento de las ventajas que proporciona las certificaciones internacionales para el mejoramiento continuo, mejora en productividad y satisfacción del cliente. Igualmente, se presentan problemas en el porcentaje de informalidad en la región, producto de la falta del cumplimiento de la ley, los controles pocos efectivos y la burocracia por parte del Estado en el trámite para la expedición de las certificaciones, aspectos que permiten el funcionamiento de las empresas sin las condiciones necesarias para el logro de procesos con calidad.

Por otra parte, desde el contexto regional se contó con la participación de Inversiones Galavis, organización en la región creada en 1918 en Villa del Rosario (La opinion, 2018) dedicada a la tostión y distribución de café, con reconocimiento por la calidad de sus productos; quien a través de la Coordinadora de Calidad Julie Chávez Vargas, relató las experiencias del proceso de certificación en normas de gestión como la ISO 9001:2008 y la ISO 22000:2005.

Esta participación fue de suma importancia, dado que es una empresa regional que cuenta con la certificación. La intervención de la coordinadora de calidad fue vital para precisar que aunque existen barreras importantes para la implementación, por la idiosincracia y cultura de los empresarios y empleados, hacerlo trae consigo ventajas para la organización y la región. No obstante, fue muy enfática, en resaltar como barreras la falta de adaptación al cambio por parte de los empleados, la inexistencia de proveedores certificados en la región y la ausencia de laboratorios en el departamento y el país para realizar pruebas exigidas en la implementación de la norma.
La cuarta ponencia realizada en el foro fue la empresa Coagronorte empresa del Norte de Santander que cultiva, produce y comercializa productos derivados del arroz (Coagronorte, s.f.). Su gerente Guillermo Infante Santos narró las experiencias en el proceso de certificación de la empresa en normas de gestión con la ISO 9001:2008. El Gerente de la empresa, resaltó que la mayoría de organizaciones tienen dificultades por los costos, la capacitación del personal, la resistencia al cambio y la inversión en infraestructura que mejoren la producción, sin embargo estas empresas desde la dirección superaron estas barreras y vienen desarrollando procesos de mejoramiento continuo, que garantizan el reconocimiento y aceptación del mercado.

Finalmente, en el Foro se dio participación a los agentes economicos de la ciudad, representados por la Camara de Comercio de Cúcuta, quien con su Gerente de Competitividad Ayllen Roció Parra Cabrera, la ponente realizó un resumen de la situación competitiva de la región incluyendo los programas y ayudas que se ofrecen desde la institución para impulsar a las empresas de la ciudad y a las personas naturales que están dispuestas a emprender nuevos negocios. De esta manera, la ponencia comenzó con el panorama actual de la región en cuanto a exportaciones que realizan las empresas.

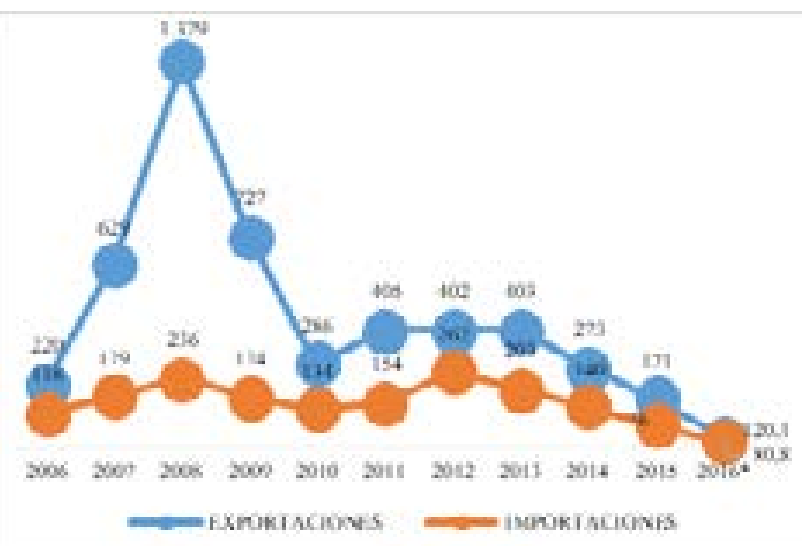

Figura 6. Panorama actual de exportaciones

Fuente (Cámara de Comercio de Cúcuta, 2018) 
La balanza comercial que dejan las exportaciones de Norte de Santander, la cual muestra el desempeño en la línea de tiempo 2006-2016 alcanzando su pico máximo de exportaciones en el año del 2008 llegando a una caída vertiginosa que se ha mantenido en los siguientes años y de la cual no se ha podido recuperar, en el año 2001 hubo un leve aumento de las exportaciones, pero después del cierre de frontera con Venezuela las exportaciones alcanzaron su desempeño más bajo manteniéndose así los últimos dos años.

Según la ponente cabe resaltar que las exportaciones de la ciudad en su mayoría se realizan de una manera intangible casi domestica las cuales no se registraban y pasaban por operaciones locales. Los principales productos exportados en el Norte de Santander, siendo el sector alimentos una de las principales empresas generadoras de exportaciones hacia países como Turquía con el 9,1\%, Guadalupe7,4\%, Brasil 7,3\% Gabón 7,2\% Estados Unidos 6,8\% a pesar de que muchos sectores de la ciudad no están registrando las exportaciones como se debería, lo cual impide tener un acercamiento más exacto de estas cifras. En este mismo sentido, la agroindustria participa con el $10 \%$ del PIB departamental (COP $\$ 1,27$ billones) siendo la palma de aceite, el cacao y la hortofrutícola sus mayores exponentes y con los cuales la cámara de comercio tiene mayor trabajo e impulso desde sus programas. Otros sectores apoyados por el área de competitividad son el de la moda, arcillas competitivas, y turismo en salud. En cuanto la última de recolección de datos para la medición competitiva de la ciudad de Cúcuta por medio de la medición doing business que enmarca el puesto de las regiones del país.

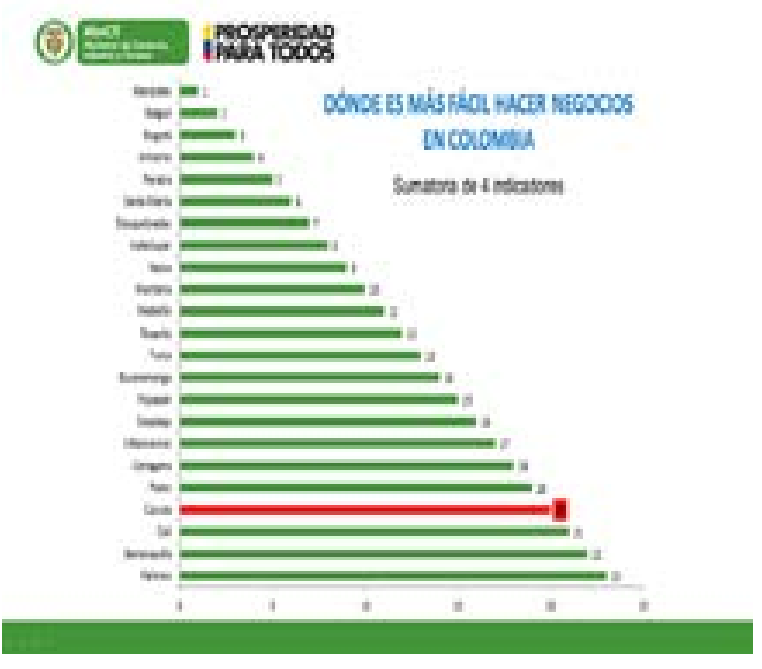

Figura 7 Región competitiva

Fuente (Consejo Privado de Competitividad, 2018)

Esta figura muestra un indicador para que los empresarios inviertan en la ciudad, lo que muestra el bajo nivel de negocios que posee la región, desde la cámara de comercio se está impulsando los sectores para mejorar estos resultados, en cuanto a los cuatro indicadores los cuales son: apertura de empresas, obtención de permisos de construcción, registro de propiedad y pago de impuestos. Para mejorar la posición competitiva de la región. Estos fueron los aspectos más importantes de la ponencia de la cámara de comercio, necesarios para indicar la situación real de la ciudad de Cúcuta en cuanto a la competitividad. Cabe resaltar que, en la intervención de la gerencia se explicaron los programas que ofrecen para incentivar a los empresarios de Norte de Santander en la creación de nuevas organizaciones y programas que apoyan la formación y establecimiento de empresas por parte de jóvenes emprendedores.

\section{c. Evaluación del diagnostico y los juicios de expertos}

La evaluación del diagnóstico y los juicios expertos se realizó por medio de una matriz de comparación, donde se medirán seis dimensiones entre las cuales está la gestión, 
la parte económica, la comunicación la parte técnica, los proveedores y la ley, respecto a la información recolectada en el objetivo uno y dos; todo esto en cuanto a las barreras que impiden el cumplimiento de las normas de gestión. La siguiente tabla reúne la matriz de comparación:

Tabla 4 Matriz de comparación expertos Foro de Calidad

\begin{tabular}{|c|c|c|}
\hline Dimensiones & Diagnostico & Juicios Expertos \\
\hline Gestión & $\begin{array}{l}\text { Barreras en: } \\
\text { - La creación del } \\
\text { sistema de gestión de } \\
\text { alimentos (creación de política } \\
\text { alcance y objetivos). } \\
\text { - Falta de compromiso } \\
\text { por parte de la alta dirección } \\
\text { de las tres empresas. } \\
\text { - En los procesos } \\
\text { que miden el desempeño de } \\
\text { las organizaciones para el } \\
\text { cumplimiento de los procesos } \\
\text { (auditorias). } \\
\text { E } \\
\text { En la planificación } \\
\text { de todas las actividades } \\
\text { que hacen parte de la } \\
\text { organización. } \\
\text { - } \\
\text { información documentada } \\
\text { necesaria. }\end{array}$ & 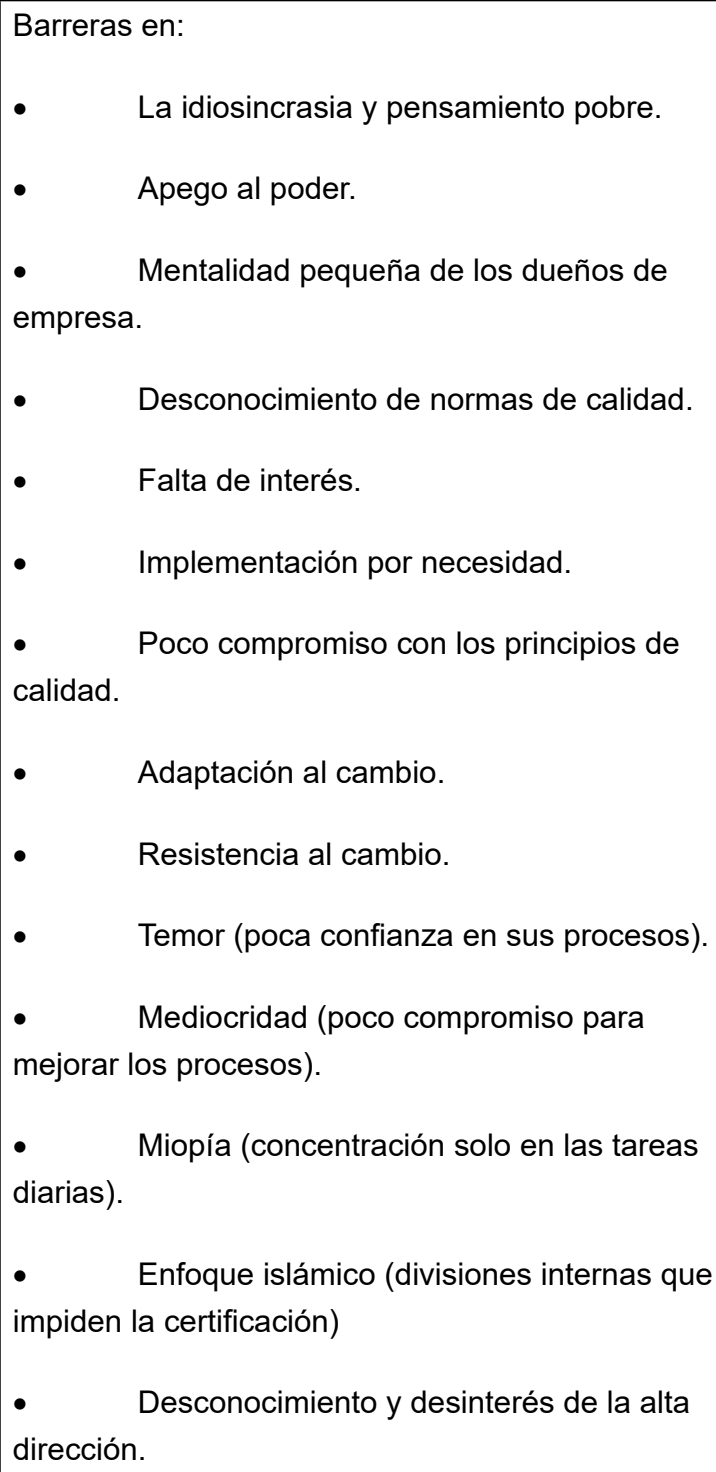 \\
\hline
\end{tabular}




\begin{tabular}{|c|c|c|}
\hline Económica & $\begin{array}{l}\text { - Falta de recursos } \\
\text { económicos para implementar } \\
\text { la norma. } \\
\text { - Inversión para } \\
\text { el mejoramiento de las } \\
\text { instalaciones. }\end{array}$ & $\begin{array}{l}\text { - } \quad \text { llusión del ahorro (no hay dinero para } \\
\text { invertir). } \\
\text { - } \quad \text { Falta de recursos económicos. } \\
\text { - Designación de recursos para la } \\
\text { adquisición de nuevas tecnologías. }\end{array}$ \\
\hline Comunicación & $\begin{array}{l}\text { - } \quad \text { Talento humano } \\
\text { capacitado. } \\
\text { - } \quad \text { Capacitación del } \\
\text { personal. } \\
\text { Trabajo en equipo. } \\
\text { - } \quad \text { Motivación y } \\
\text { evaluación del personal. } \\
\text { - } \quad \text { Educación de } \\
\text { trabajadores y alta dirección }\end{array}$ & $\begin{array}{l}\text { - } \\
\text { - } \quad \text { Talento humano capacitado. } \\
\text { - } \quad \text { Trabajo en equipo. } \\
\text { Motivación y evaluación del personal. } \\
\text { - } \quad \text { Educación de trabajadores y alta } \\
\text { dirección }\end{array}$ \\
\hline Técnica & $\begin{array}{l}\text { - Instalaciones muy } \\
\text { pequeñas que impiden el } \\
\text { cumplimiento de disposiciones } \\
\text { técnicas. } \\
\text { - En identificación y } \\
\text { evaluación de peligros. } \\
\text { - Falencias para } \\
\text { establecimiento de sistemas } \\
\text { de trazabilidad. } \\
\text { - } \quad \text { Procedimientos } \\
\text { documentados que garanticen } \\
\text { la estandarización de } \\
\text { procesos. } \\
\text { - } \quad \text { Registros adecuados }\end{array}$ & $\begin{array}{l}\text { - } \\
\text { - } \quad \text { Fruebas de laboratorio. } \\
\text { - }\end{array}$ \\
\hline Proveedores & $\begin{array}{l}\text { - Proveedores } \\
\text { certificados } \\
\text { - } \quad \text { Control de } \\
\text { proveedores en todas las } \\
\text { etapas productivas }\end{array}$ & Búsqueda de proveedores certificados \\
\hline Ley & $\begin{array}{l}\text { - En el cumplimiento del } \\
100 \% \text { de los aspectos de } \\
\text { ley }\end{array}$ & $\begin{array}{l}\text { - Informalidad de la región. } \\
\text { - } \quad \text { Cumplimiento escaso de la ley. } \\
\text { - Controles poco efectivos por parte del gobierno. }\end{array}$ \\
\hline
\end{tabular}




\section{CONCLUSIONES}

El diagnóstico realizado en la industria cárnica sirvió como parámetro de inicio para detectar las principales falencias de este sector, confirmando la problemática planteada en el anteproyecto de investigación, de esta manera se resalta la gran diferencia entre los procesos desarrollados en las empresas con los requisitos exigidos por la norma de inocuidad alimentaria. Se evaluaron aspectos de las certificaciones internacionales, los cuales sirvieron como fuente primordial de información para verificar las practicas desempeñadas por las plantas de producción de derivados cárnicos. Se encontraron resultados con un desempeño bajo para sistemas de gestión de inocuidad alimentaria; lo que indica que el sector desconoce las normas que ayudan a mejorar los procesos de inocuidad y los beneficios que trae consigo la implementación. Con el diagnóstico se pudo observar que el comportamiento de las empresas de este sector en cuanto a la norma es muy similar, siendo este un factor preponderante para concluir que la competencia como empresas de una misma sección se debe basar en el mejoramiento de los procesos de inocuidad con el fin de garantizar en lo consumidores seguridad y variedad de elección.

A partir de estas observaciones encontradas mediante el diagnóstico es importante incluir a todos los sectores de la sociedad para generar soluciones de los problemas que afectan a la industria; desde la academia y por medio de la investigación se pretendía encontrar las barreras que impiden el cumplimiento de la norma, con el fin de mejorar como un todo no solo evidenciando las fallas en la producción, sino por lo contrario realizando la labor de encontrar esas dificultades para que sea más fácil la implementación de normas de calidad en un futuro a las empresas de la región.
Así mismo se buscó la participación de expertos en sistemas de gestión con el fin de abarcar una mayor visión de las barreras que impiden el cumplimiento en las normas de calidad, se puede señalar que para la investigación fue de gran aporte todos los juicios mencionados tanto por asesores nacionales e internacionales y por empresas que han participado en procesos de certificación, con lo cual se puede concluir que existe gran similitud en lo expresado por los ponentes ya que se encontraron barreras similares como la resistencia al cambio, desconocimiento y falta de compromiso por los dueños de las empresas al igual que la idiosincrasia de la región la cual impide el mejoramiento de las empresas de la ciudad.

La participación de empresarios en el foro de calidad sirvió también para ampliar la visión respecto a las normas de calidad dando paso al mejoramiento de los procesos desempeñados en las organizaciones. Por otra parte, la participación de la cámara de comercio de Cúcuta hace notar, que la ciudad se encuentra en mal estado en cuanto a competitividad siendo este sector parte fundamental de la pequeña industria cucuteña, y que mediante la implementación de normas de gestión se puede mejorar la situación que nos afecta, generando las posibilidades de nuevos mercados siendo fundamentales los certificados de calidad. De esta manera se confirmó que el sector posee una participación nula en las exportaciones de productos de la ciudad, pero a nivel regional esta industria es un gran motor de desarrollo para la región. Cabe resaltar la participación de estudiantes de diferentes universidades que, con sus aportes y participación en el foro enriquecieron de forma valiosa la investigación y sobre todo las debilidades de la región en cuanto a la certificación de normas de calidad.

Las barreras encontradas en la industria son creadas por situaciones específicas del contexto actual en el que se encuentra la región, al 
evaluar el diagnóstico y los juicios expertos se encontraron similitudes con los procesos que pasaron empresas de alimentos de la ciudad que han emprendido la labor de certificarse en normas de calidad. Siendo estas las generadoras de los cuatro grupos de barreras las cuales se clasificaron en: gestión, económicas, técnicas y de comunicación, agrupando todos los escenarios que llegan a causar impedimentos para la implementación de normas de calidad en la industria cárnica de bovino de la ciudad de Cúcuta. Fue fundamental para la industria conocer estas dificultades puesto que ellas son el factor principal del atraso de las empresas en cuanto a normas de gestión, y es el posible comienzo del mejoramiento de los procesos.

\section{REFERENCIAS BIBLIOGRÁFICAS}

\section{JACOME. (2017). CVBYU. CUCUTA.}

Departamento Nacional de Planeación. (2004). Cadenas Productivas protección. (D. N. Planeación, Ed.) Bogotá, Cundinamarca, Colombia: Departamento Nacional de Planeación.

Fedegan. (2019). Consumo. Recuperado el 06 de 2020, de Fedegan: https://www. fedegan.org.co/estadisticas/consumo-0

Guarin, A. (2008). Carne De Cuarta Para Consumidores De Cuarta. Revista de Estudios Sociales, 29, 104-119.

Gobierno de Colombia. (2019). Pacto por el crecimiento y la generación de empleo del sector cárnico. Bogotá.

Ministerio de Agricultura. (2019). Cadéna cárnica bovina. Recuperado el 06 de 2020, de Ministerio de Agricultura: https:// sioc.minagricultura.gov.co/Bovina/ Documentos/2019-06-30\%20Cifras $\% 20$ Sectoriales.pdf
Gobierno de Colombia. (2019). Pacto por el crecimiento y la generación de empleo del sector cárnico. Sectorial, Gobierno de Colombia, Bogotá.

Carro, R., \& Gonzalez, D. (s.f.). Norma HACCP Sistema de análisis de riesgos críticos de Control. Obtenido de http://nulan. mdp.edu.ar/1616/1/11_normas_haccp. pdf

Hernández, R. F. (1998). Metodología de la Investigación. Mexico, Mexico: McgrawHill.

Lucio, R. H. (2003). Metodología de la Investigación. Mexico: McGraw-Hill.

HERNANDEZ SAMPIERI, R. F. (2003). Metodología de la Investigación. Mexico: MacGraw-Hill.

Arias, F. (2006). El proyecto de investigación. Caracas: Episteme.

Otzen, T., \& Manterola, C. (2017). Técnicas de mestreo sobre una población de estudio. Internacional Journal of Morphology, 35, 227-232.

ICONTEC. (2005). NORMA TECNICA COLOMBIANA. Bogotá: INCONTEC.

Heras, I., Arana, G., Camison, C., Casadesus, M., \& Martiarena, A. (2008). Gestion de la calida y competitividad. Bilbao: Deusto.

Heizer, R. (2007). Adminitración de la Producción. Mexico: Pearson Educación.

Ortiz, A., \& Martinez, M. (2011). Inocuidad Alimentaria: Panorama en Colombia. Conexión Agropecuaria , 1, 37-44.

Bryan , F. (1992). Evaluación por analisis de peligros en puntos criticos de control. Ginebra. 
Slim, C. (2018). Crea mi empresa. Recuperado el 01 de 08 de 2020, de https:// crearmiempresa.es/article-consejosempresarios-carlos-slim-86747609.html

Coagronorte. (s.f.). Coagronorte. Recuperado el 07 de 08 de 2020, de https://www. coagronorte.com.co/

Organización Internacional de Normalización. (2015). Sistemas de gestión de la calidad - Fundamentos y vocabulario. Incontec.

Ministerio de Salud. (4 de Mayo de 2007). Decreto 1500 de 2007. Recuperado el 30 de julio de 2020, de Ministerio de Salud: https://www.minsalud.gov. $\mathrm{co} /$ sites/rid/Paginas/freesearchresults. asp $\mathrm{x}$ ? $\mathrm{k}=\& \mathrm{k}=$ decreto $\% 201500 \% 20$ de $\% 202007$

Coneo, M. (30 de 09 de 2019). Conmemoración Anual del Sector. Recuperado el 05 de 05 de 2020, de Agronegocios: https://www.agronegocios.co/ferias/ conmemoracion-anual-al-sectorque-aporta-16-al-pib-nacional-losganaderos-2915639

Portafolio. (29 de 01 de 2020). Exportaciones de carne siguen creciendo a pesar de la aftosa. Recuperado el 06 de 05 de 2020, de https://www.portafolio.co/economia/ exportaciones-de-carne-siguencreciendo-a-pesar-de-la-aftosa-537596

Asmar, S. (16 de 03 de 2020). EN 2020, COLOMBIA YA EXPORTÓ MÁS DE 4.500TONELADASDE CARNEBOVINA A OCHO PAÍSES. La Republica.

Insituto Agropecuario Colombiano. (06 de Febrero de 2020). Colombia recupera su estatus sanitario como país libre de fiebre aftosa con vacunación. Recuperado el 07 de 05 de 2020, de https://www.ica.gov.co/noticias/ colombia-recupera-estatus-pais-libreaftosa

Procolombia. (2015). El mundo invierte en Colombia. Recuperado el 08 de 05 de 2020, de https://www. inviertaencolombia.com.co/images/ Adjuntos/SECTOR-CARNICOS2016. pdf

Departamento Nacional de Planeación. (01 de 07 de 2020). Cadenas Productivas. Recuperado el 07 de 2020, de Departamento Nacional de Planeación: https://www.dnp.gov.co/programas/ desarrollo-empresarial/Paginas/ analisis-cadenas-productivas.aspx

Nieto, V., \& Ramirez, N. (2018). Cadenaproductiva de Carnes y Productos Cárnicos Estructura, Comercio Internacional $y$ Protección. Departamento Nacional de Planeación. Bogotá: Departamento Nacional de Planeación.

Departamento Nacional de Planeación. (2018). Cadena productiva de Carnes y Productos Cárnicos Estructura, Comercio Internacional y Protección. Departamento Nacional de Planeación. Bogotá: Departamento Nacional de Planeación.

Miniserio de Agricultura y Desarrollo Rural. (2010). Planes de desarrollo para cuatro sectores clave de la agroindustria de Colombia. Miniserio de Agricultura y Desarrollo Rural. Bogotá: Miniserio de Agricultura y Desarrollo Rural. 
Ministerio de Protección Social. (04 de 05 de 2007). Decreto 1500 de 2007. Recuperado el 05 de 06 de 2020, de https://www.minsalud.gov.co/sites/rid/ Lists/BibliotecaDigital/RIDE/DE/DIJ/ Resolucion\%202905\%20de\%202007. PDF

República, P. d. (1997). Decreto 3075 dee 1997. Recuperado el 18 de 06 de 2020, de https://www.minsalud.gov.co/ Normatividad_Nuevo/DECRETO\%20 3075\%20DE\%201997.pdf

Ministerio de Protección Social. (26 de 06 de 2009). Decreto 2380 de 2009. Recuperado el 20 de 06 de 2020, de ttps://www.ica.gov.co/getattachment/ a b a 189 b 4 - f 7 c 9 - 4 c 75 - b e 96 f6d7d27b5bce/2009D2380.aspx

Ministerio de la Protección Social. (22 de 08 de 2007). Resolución 2905 de 2007. Recuperado el 20 de 06 de 2020, de https://www.minsalud.gov.co/sites/rid/ Lists/BibliotecaDigital/RIDE/DE/DIJ/ Resolucion\%202905\%20de\%202007. PDF

Ministerio de la Protección Social. (22 de 07 de 2013). RESOLUCIÓN 2674 DEL 2013. Recuperado el 20 de 06 de 2020, de https://www.minsalud.gov.co/sites/rid/ Lists/BibliotecaDigital/RIDE/DE/DIJ/ resolucion-2674-de-2013.pdf

ISO. (2018). ISO . Recuperado el 20 de 06 de 2020, de https://www.iso.org/obp/ ui\#iso:std:iso:22000:ed-2:v2:es

Iso. (2015). ISO. Recuperado el 20 de 06 de 2020, de https://www.iso.org/obp/ ui/\#iso:std:iso:9001:ed-5:v1:es

Velasquez, E. (2009). Calidad de la gestion administrativa de la facultad de ciencias economicas y administrativas de la Universidad Nacional de. púa, Calidad de la gestion administrativa de la facultad de ciencias economicas y administrativas de la Universidad Nacional de.

INCONTEC. (2019). Sistemas de gestiòn de la Inocuidad de los alimentos. Bogotá: INCONTEC.

INCONTEC. (2019). Sisema de Gestión de la Inocuidad de los alimentos. Bogotá: INCONTEC.

Hermann Keyserling. (2010). Lidrezgo en los sistemas de gestión. Recuperado el Julio de 2020, de Calidad y Gestión: https:// calidad-gestion.com.ar/boletin/57_ liderazgo_sistemas_de_gestion.html

INCONTEC. (2019). Sistema de Gestión de la Inocuidad . Bogotá: INCONTEC.

Organización Panamericana de Salud. (s.f.). Analisis de Peligros y puntos criticos de control (HACCP). Recuperado el 08 de 2020, de Organización Panamericana de Salud: https://www.paho.org/hq/ dmdocuments/2017/food-safety-hacppcha-analisis-peligros-puntos-criticoscontrol.pdf

La opinion. (17 de 07 de 2018). Café Galavis llega a 100 años de historia. Recuperado el 02 de 08 de 2020, de https://www.laopinion. com.co/historicos/cafe-galavis-llega100-anos-de-historia-158471

Cámara de Comercio de Cúcuta. (2018). Balanza Comercial. Cúcuta: Cámara de Comercio de Cúcuta.

Consejo Privado de Competitividad. (2018). Indice Departamental de Competitividad. Bogotá: Consejo Privado de Competitividad. 